\title{
Full panretinal photocoagulation and early vitrectomy improve prognosis of retinal vasculitis associated with tuberculoprotein hypersensitivity (Eales' disease)
}

\author{
A M Abu El-Asrar, S A Al-Kharashi
}

Background/aims: Eales' disease is an uncommon vasoproliferative retinal disease affecting otherwise healthy young men that is characterised by obliterative retinal periphlebitis, with sequelae such as recurrent vitreous haemorrhage and traction retinal detachment. This study was undertaken to determine whether visual prognosis of Eales' disease could be improved by appropriate medical and surgical treatment.

Methods: The authors retrospectively studied 30 patients (46 eyes) who were treated from 1992 to 2001 . Recorded data included patient age, sex, race, medical history, medications, results of the ophthalmological examination, results of diagnostic laboratory evaluation, and details of systemic and surgical treatments. The mean follow up was 10.6 months.

Results: 19 patients (23 eyes) who presented with active periphlebitis received systemic steroids and antituberculous therapy. Extensive full panretinal photocoagulation was performed in 21 eyes that presented with new vessel formation and peripheral capillary closure with or without vitreous haemorrhage. Vitrectomy and endolaser panretinal photocoagulation was necessary in 15 eyes, for severe non-clearing vitreous haemorrhage in 11 eyes and vitreous haemorrhage with traction retinal detachment in four eyes. Complete regression of the disease was achieved in all eyes. Vitrectomy resulted in a significant visual improvement with 14 of the 15 eyes $(93.3 \%)$ achieving $\geqslant 20 / 200$ visual acuity. Overall, the distribution of visual acuities among eyes improved from presentation to final follow up, with $36.4 \%$ of eyes having $20 / 40$ or better acuity at presentation compared with $63.6 \%$ of eyes by final follow up. Conclusions: These results suggest that aggressive treatment of Eales' disease with systemic steroids and antituberculous therapy, full panretinal photocoagulation and early vitrectomy, when necessary, may result in improving the anatomic and visual outcome.

n 1880 Henry Eales described a syndrome of recurrent retinal and vitreous haemorrhage in young men, associated with constipation and epistaxis. ${ }^{1}$ Currently, Eales' disease is considered to be an idiopathic inflammatory venous occlusion that primarily affects the peripheral retina. Retinal changes include perivasculitis, mainly periphlebitis, and peripheral non-perfusion. This inflammation induced vascular occlusion can lead to a proliferative vascular retinopathy, with sequelae such as recurrent vitreous haemorrhage and traction retinal detachment. ${ }^{2-5}$

Eales' disease appears most commonly to affect healthy young adults in the third and fourth decades of life. ${ }^{6}$ The disease is prevalent in India, Pakistan, and Afghanistan, ${ }^{6}$ and is strongly associated with tuberculoprotein hypersensitivity. ${ }^{237}$
Some patients diagnosed with Eales' disease have had concurrent active pulmonary tuberculosis. ${ }^{38}$ Although the clinical characteristic and natural course of Eales' disease are well known, its aetiopathogenesis is not yet well understood. Of the several aetiologies proposed, most favoured are tuberculosis and hypersensitivity to tuberculoprotein. ${ }^{5}$ Recently, Mycobacterium tuberculosis complex DNA was detected in vitreous fluid samples of Eales' disease patients using polymerase chain reaction. ${ }^{9}$ Another study, demonstrated the presence of Mycobacterium tuberculosis DNA by nested polymerase chain reaction technique in epiretinal membrane specimens from patients with Eales' disease. ${ }^{10}$ Bacteriological examination of vitreous fluid samples did not reveal the presence of acid fast bacilli. ${ }^{9}$ In addition, Biswas et $a l^{11}$ found statistically significant higher phenotype frequencies of HLA B5 (B51), DR1, and DR4 in patients with Eales' disease compared with healthy people. It is hypothesised that individuals with the HLA predisposition may develop retinal vasculitis as a result of a cell mediated immunological tissue damage triggered by a sequestered Mycobacterium tuberculosis antigen in an inactive form and clinically present as Eales' disease. ${ }^{10}$

In this study, we have reviewed the records of 30 patients with Eales' disease (46 eyes) who were treated in our institution to determine the anatomical and visual outcome.

\section{PATIENTS AND METHODS}

The medical records of 30 consecutive patients (46 eyes) who received a diagnosis of Eales' disease were reviewed

Table 1 Diagnostic studies performed on patients with Eales' disease

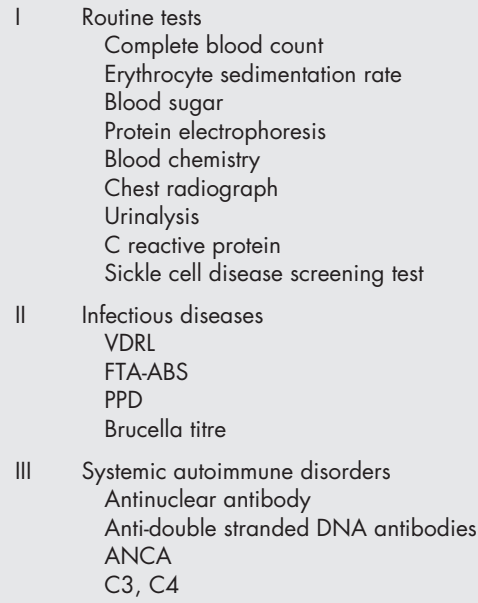

VDRL = Venereal Disease Research Laboratory; FTA-ABS = fluorescent treponemal antibody absorption test; PPD = purified protein derivative; ANCA = antineutrophil cytoplasmic antibody. 


\begin{tabular}{|c|c|}
\hline Findings & No of eyes (\%) \\
\hline Sclerotic vessels & $38(82.6)$ \\
\hline Neovascularisation & $32(69.6)$ \\
\hline Vitreous haemorrhage & $29(63)$ \\
\hline Active periphlebitis & $23(50)$ \\
\hline Traction retinal detachment & $8(17.4)$ \\
\hline Chorioretinal scars & $2(4.3)$ \\
\hline Multifocal choroiditis & $1(2.2)$ \\
\hline Serous retinal detachment & $1(2.2)$ \\
\hline
\end{tabular}

retrospectively. Patients were regarded as having Eales' disease if they had unilateral or bilateral obliterative retinal vasculitis affecting the veins without a known and definite cause for retinal vascular disease (for example, branch vein occlusion, diabetes, sarcoidosis, or identifiable systemic autoimmune or neurological disorders) and associated with tuberculoprotein skin positivity.

At presentation, all the patients had had a full medical and ophthalmological examinations. This included a history and a physical, neurological, and ophthalmological examination and laboratory tests. The list of laboratory tests is included in Table 1. Ophthalmological evaluation included determination of visual acuity, applanation tonometry, slit lamp examination of the anterior segment, and direct and indirect ophthalmoscopy, including examination of the posterior and peripheral retina with a Goldmann three mirror contact lens after pupillary dilatation, fundus photographs, and intravenous fluorescein angiography in eyes with clear media.

The following medical and demographic data were recorded: patient age, sex, race, medical history, medications on presentation, results of the ophthalmological examination, and the results of diagnostic laboratory evaluation. In addition, details of systemic and surgical treatment were noted.

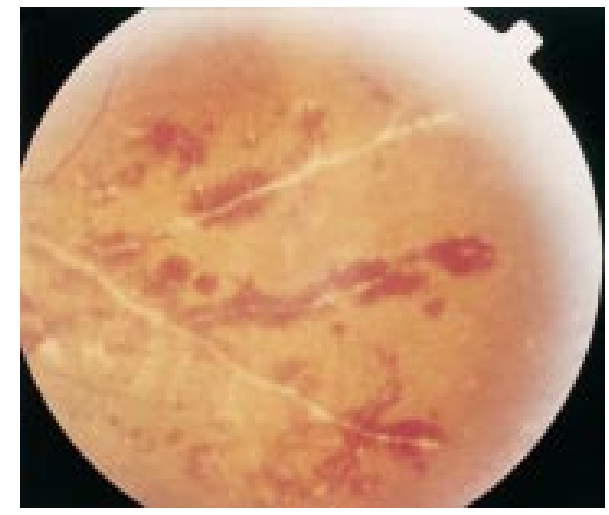

Figure 3 Fundus photograph of the left eye. The temporal retina demonstrates perivenous sheathing with intraretinal haemorrhage.

\section{RESULTS}

Eales' disease was diagnosed in 30 patients (46 eyes) seen at the retina clinic, King Abdulaziz University Hospital, Riyadh, between 1992 and 2001. Twenty eight patients were male $(93.3 \%)$ and two were female $(6.7 \%)$. The mean age of patients was 30.8 (SD 5) years (range 22-45 years). Eleven were from India, nine from Bangladesh, four from Pakistan, and six were Saudi. The disease was bilateral in 16 patients and unilateral in 14 patients.

The posterior segment findings noted at presentation or during pars plana vitrectomy are presented in Table 2 . The most common finding was sclerotic vessels $(82.6 \%)$, followed by retinal neovascularisation (69.6\%) (Fig 1), vitreous haemorrhage (63\%) (Fig 2), active periphlebitis (50\%) (Fig 3), traction retinal detachment (17.4\%) (Fig 4), chorioretinal scars $(4.3 \%)$, multifocal choroiditis $(2.2 \%)$, and serous retinal detachment $(2.2 \%)$. Patients who had active periphlebitis showed moderate vitreous infiltrate and intraretinal haemorrhage.
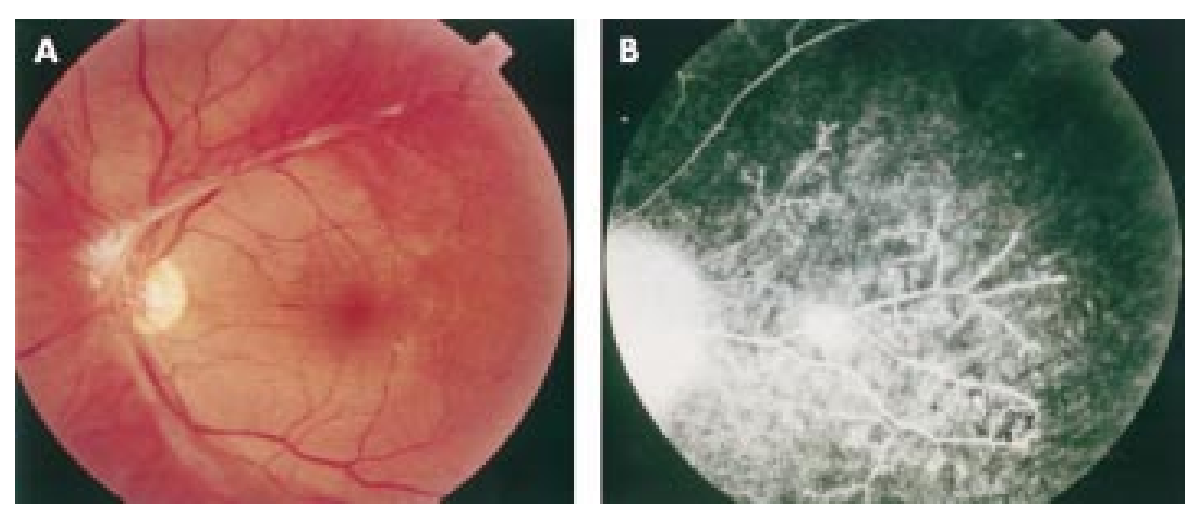

Figure 1 (A) Fundus photograph of the left eye showing a large patch of fibrovascular proliferation.

(B) Fluorescein angiography showing filling of the fibrovascular

proliferation with abnormal leakage.

Note retinal non-perfusion peripheral to fibrovascular proliferation.
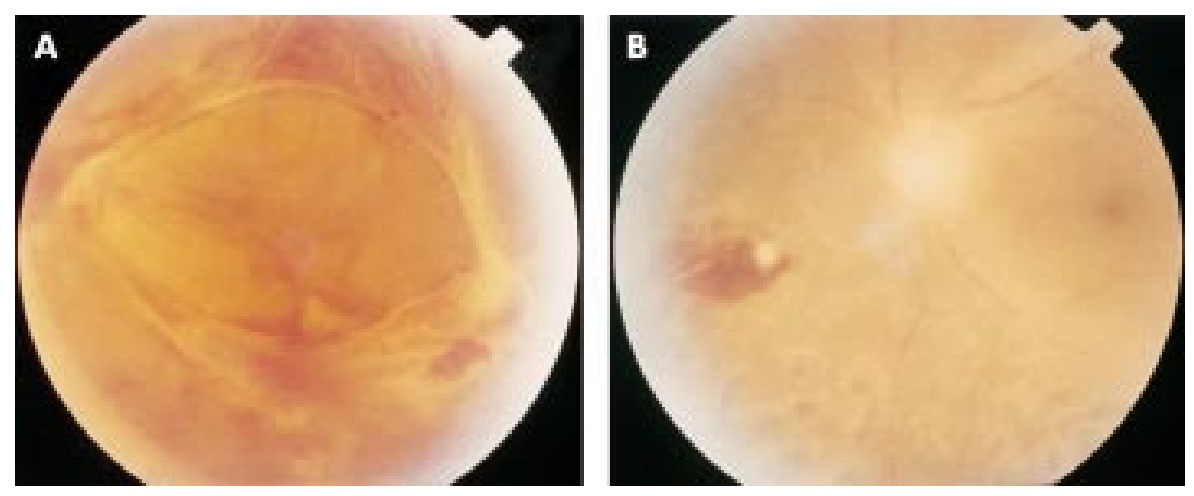

Figure 2 (A) Vitreous haemorrhage and active fibrovascular proliferation in a patient with Eales' disease. (B) 1 month after pars plana vitrectomy and endolaser panretinal photocoagulation. 


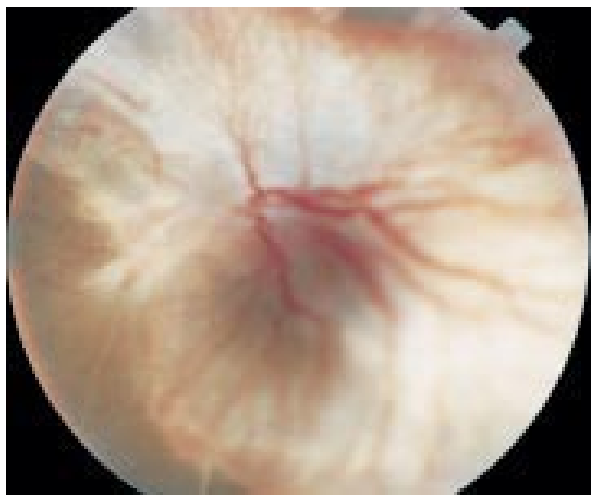

Figure 4 Severe traction retinal detachment in a patient with Eales' disease. The patient had evidence of active spinal tuberculosis.

All the patients had strongly positive tuberculin skin tests (five tuberculin units). The area of induration ranged from 15 $\mathrm{mm}$ to $60 \mathrm{~mm}$ (mean 25.4 (SD 10.8) $\mathrm{mm}$ ) at 48 hours. Chest radiographs were normal, and one patient who presented with severe bilateral traction retinal detachment had evidence of active spinal tuberculosis. None of the patients had evidence of other systemic diseases such as diabetes, hypertension, blood dyscrasias, haemoglobulinopathies, sarcoidosis, or systemic lupus erythematosus.

Fluorescein angiography delineated the regional vascular abnormalities. These included leakage of dye due to breakdown of the inner blood retinal barrier, staining of the blood vessel wall with fluorescein, capillary non-perfusion, capillary dilatation, arteriovenular shunts, and high degree of fluorescein extravasation through the pathological neovascularisation (Fig 1).

Nineteen patients (23 eyes) who presented with active periphlebitis received systemic prednisone $(1 \mathrm{mg} / \mathrm{kg} / \mathrm{day}$ until a clinical effect was seen then a slow reduction was established) and antituberculous (isoniazide $300 \mathrm{mg} /$ day, ethambutol $15 \mathrm{mg} / \mathrm{kg} /$ day, rifampin $600 \mathrm{mg} /$ day, and pyrazinamide $2 \mathrm{mg} /$ day) drugs for at least 6 months. Twenty one eyes that showed new vessel formation and peripheral capillary closure with or without vitreous haemorrhage were treated with full panretinal photocoagulation starting with the inferior retinal quadrants to reduce the risk of vitreous haemorrhage which could prevent continuation of the treatment in these areas. Vitrectomy was performed in a total of 15 eyes, for severe non-clearing vitreous haemorrhage in 11 eyes, and vitreous haemorrhage with traction retinal detachment in four eyes. Temporary silicone oil tamponade was used in two eyes with traction retinal detachment, and relaxing retinectomy was required in one of these two eyes. Encircling scleral buckles were placed in all eyes with traction retinal detachment. Endolaser panretinal photocoagulation was used in all eyes that underwent vitrectomy. Three eyes had severe traction retinal detachment that was judged to be inoperable, and one eye had no light perception at presentation because of traction retinal detachment.

Follow up data were obtained on 28 patients (44 eyes). The remaining two patients (two eyes) were lost to follow up. Of these 28 patients, mean follow up was 10.6 (SD 5.1) months (range 4-24 months). The range of initial and final visual acuities is set out in Table 3. Complete regression of the disease was achieved in all eyes. Full panretinal photocoagulation resulted in complete involution of neovascularisation. Vitrectomy resulted in retinal reattachment and in a vitreous cavity which stayed clear until the last follow up, and the visual acuity improved significantly (Fig 2). Table 4 shows the range of initial and final visual acuities in eyes treated with vitrectomy, in eyes treated with panretinal photocoagulation, and in eyes that had only active periphlebitis at presentation.

\section{DISCUSSION}

Eales' disease is in essence a diagnosis of exclusion, and other systemic disease such as diabetes, hypertension, blood dyscrasias, haemoglobulinopathies, sarcoidosis, or systemic lupus erythematosus should be ruled out. The earliest stages of the disease are marked by low grade periphlebitis. Later, the disease progresses to increasing areas of non-perfusion of the capillaries of the peripheral retina. With progression of the disease, retinal capillary closure extends contiguously in a posterior direction. With increasing retinal non-perfusion and ischaemia, neovascularisation can appear, usually at the junction of perfused and non-perfused retina. The patient remains unaware of these early changes. However, when bleeding from the abnormal new blood vessels occurs, the patient can notice "floating spots" or blurred vision. Ultimately, Eales' disease can progress to functional loss of the eye through traction retinal detachment.

Steroids should be of benefit in patients with tuberculin hypersensitivity and retinal periphlebitis. ${ }^{12}{ }^{13}$ Rosen and coworkers reported seven patients with retinal vasculitis in the presence of a strongly positive Mantoux test. The characteristic features were a moderate vitreous infiltrate, severe periphlebitis, and a marked tendency to peripheral retinal capillary closure leading to new vessel formation. The patients were treated with systemic corticosteroids, with or without antituberculous therapy. ${ }^{12}$ All cases of retinal vasculitis resolved with treatment. Reactivation of miliary tuberculosis

Table 3 Initial visual acuity plotted against final visual acuity for 44 eyes. The shaded squares represent the position at which initial and final visual acuities are equal. The numbers above the shaded squares indicate an improvement in visual acuity

\begin{tabular}{|c|c|c|c|c|c|c|c|c|c|c|c|}
\hline \multirow[b]{2}{*}{ Final VA } & \multicolumn{10}{|c|}{ Initial visual acuity (VA) } & \multirow[b]{2}{*}{ Tota } \\
\hline & NLP & LP & $\mathrm{HM}$ & $\mathrm{CF}$ & $20 / 200$ & $20 / 100$ & $20 / 60$ & $20 / 40$ & $20 / 30$ & $20 / 20$ & \\
\hline $20 / 20$ & - & - & 1 & 2 & 2 & 1 & - & 4 & 4 & 6 & 20 \\
\hline $20 / 30$ & - & - & - & 1 & 2 & - & 1 & - & - & 1 & 5 \\
\hline $20 / 40$ & - & - & - & - & - & 1 & 1 & 1 & - & - & 3 \\
\hline $20 / 60$ & - & - & 1 & 3 & 1 & - & - & - & - & - & 5 \\
\hline $20 / 100$ & - & 1 & - & 1 & - & - & - & - & - & - & 2 \\
\hline $20 / 200$ & - & 1 & 1 & 1 & - & - & - & - & - & - & 3 \\
\hline CF & - & - & - & 2 & - & - & - & - & - & - & 2 \\
\hline HM & - & - & 1 & - & - & - & - & - & - & - & 1 \\
\hline LP & - & 2 & - & - & - & - & - & - & - & - & 2 \\
\hline NLP & 1 & - & - & - & - & - & - & - & - & - & 1 \\
\hline Total & 1 & 4 & 4 & 10 & 5 & 2 & 2 & 5 & 4 & 7 & 44 \\
\hline
\end{tabular}


Table 4 Initial and final visual acuities

\begin{tabular}{lllllllll}
\hline & \multicolumn{2}{l}{ Initial visual acuity (\%) } & & & \multicolumn{3}{l}{ Final visual acuity (\%) } \\
\cline { 2 - 3 } Visual acuity & $\operatorname{PPV}^{*}(n=15)$ & $\operatorname{PRPt}(n=19)$ & $\operatorname{RV}(n=6)$ & & $\operatorname{PPV}(n=15)$ & $\operatorname{PRP}(n=19)$ & $\operatorname{RV}(n=6)$ \\
\hline LP-CF & $13(86.7)$ & $2(10.5)$ & - & & $1(6.7)$ & $1(5.3)$ & - \\
$20 / 200-20 / 60$ & $2(13.3)$ & $7(36.8)$ & - & & $10(66.7)$ & - & - \\
$20 / 40-20 / 20$ & - & $10(52.7)$ & $6(100)$ & & $4(26.6)$ & $18(94.7)$ & $6(100)$ \\
\hline
\end{tabular}

$\mathrm{LP}=$ light perception; $\mathrm{CF}=$ counting fingers; $P P V=$ eyes underwent pars plana vitrectomy; $P R P=$ eyes treated with panretinal photocoagulation (two patients were lost to follow up); RV = eyes presented with only active periphlebitis.

*Three eyes had active periphlebitis.

$\dagger 13$ eyes had active periphblebitis, 9 eyes had vitreous haemorrhage, 2 eyes had chorioretinal scars, and

one eye had multifocal choroiditis and serous retinal detachment.

with choroidal tubercles occurred in one patient treated with corticosteroids alone. This underscores the risk of systemic corticosteroids in PPD positive patients who are not given concomitant antituberculous drugs. Therefore, we strongly suggest that retinal periphlebitis with strongly positive PPD test requires the use of systemic steroids and appropriate antituberculous therapy even in the absence of active systemic disease to avoid reactivation of the systemic illness.

Eales' disease is a vasoproliferative retinal disease characterised by progressive peripheral retinal capillary closure resulting in increasing retinal non-perfusion, ischaemia, and neovascularisation. In fact, its progression and clinical appearance are similar to other vasoproliferative diseases of the retina such as diabetic and sickle cell retinopathies. These similarities suggest that treatment by panretinal photocoagulation should be effective in patients with capillary dropout and neovascularisation. Magargal and coworkers have demonstrated the efficacy of panretinal photocoagulation in controlling the neovascularisation and achieving complete regression of the disease. ${ }^{14}$ In this series, complete regression of vasoproliferative disease was achieved after extensive full panretinal photocoagulation. Therefore, we strongly recommend immediate laser photocoagulation to rapidly achieve a full, extensive panretinal photocoagulation before vitreous haemorrhage and fibrovascular proliferation and traction can develop.

In the course of Eales' disease treatment, vitrectomy frequently is required. In this series, 15 eyes (32.6\%) required vitrectomy for unresolving vitreous haemorrhage in 11 eyes and vitreous haemorrhage with traction retinal detachment in four eyes. During vitrectomy, adequate endolaser photocoagulation is necessary to reduce the risk of recurrent bleeding. Vitrectomy with posterior hyaloid dissection should not be delayed for patients with unresolving vitreous haemorrhage and active fibrovascular proliferation because progressive fibrovascular proliferation while waiting for vitreous haemorrhage to clear may cause traction retinal detachment. In this series, vitrectomy and endolaser photocoagulation resulted in a permanently clear vitreous cavity in all 15 eyes, and the visual acuity improved significantly, reaching 20/200 or greater in 14 of the 15 eyes (93.3\%). Similarly, Shanmugan et $a l^{15}$ reported good visual results following vitrectomy in majority of patients with complications of Eales' disease. The distribution of visual acuities among eyes improved from presentation to final follow up, with $43.2 \%$ of eyes having counting fingers or less at presentation as compared with $13.6 \%$ of eyes by final follow up, and $36.4 \%$ of eyes having $20 / 40$ or better acuity at presentation compared with $63.6 \%$ of eyes by final follow up.

The practising ophthalmologist who is evaluating a patient with suspected Eales' disease should begin by ruling out the presence of other disorders with appropriate tests as mentioned. Once the ophthalmologist is reasonably certain that the patient has Eales' disease, the presence of active pulmonary tuberculosis should be excluded and then the patient's tuberculoprotein hypersensitivity status determined. Patients presenting with active retinal periphlebitis in the presence of positive PPD test should be given systemic steroids and appropriate antituberculous therapy to avoid reactivation of the systemic illness. New vessel formation associated with retinal vasculitis and capillary closure responds to full panretinal photocoagulation. Early vitrectomy and adequate endolaser photocoagulation should be considered in eyes with non-resolving vitreous haemorrhage associated with active fibrovascular proliferation. The good visual results following vitrectomy in Eales' disease may be due to the peripheral nature of the vascular changes.

\section{ACKNOWLEDGEMENT}

The authors thank Ms Connie B Unisa-Marfil for her secretarial work.

\section{Authors' affiliations}

A M Abu El-Asrar, S A Al-Kharashi, Department of Ophthalmology, College of Medicine, King Saud University, Riyadh, Saudi Arabia

Correspondence to: Professor Dr Ahmed M Abu El-Asrar, Department of Ophthalmology, King Abdulaziz University Hospital, Airport Road, PO Box 245, Riyadh 11411, Saudi Arabia; abuasrar@ksu.edu.sa

Accepted for publication 19 June 2002

\section{REFERENCES}

1 Eales H. Primary retinal hemorrhage in young men. Ophthalmol Rev $1882 ; 1: 41-6$.

2 Elliot AJ, Harris GS. The present status of the diagnosis and treatment of periphlebitis retinae (Eales' disease). Can J Ophthalmol 1969;4:117-22.

3 Renie WA, Murphy RP, Anderson KC, et al. The evaluation of patients with Eales' disease. Retina 1983;3:243-8.

4 Theodossiadis $\mathbf{G}$. Fluorescein angiography in Eales' disease. Am J Ophthalmol 1970;69:271-7.

5 Das T, Biswas J, Kumar A, et al. Eales' disease. Indian J Ophthalmol 1994;42:3-18.

6 Helm CJ, Holland GN. Ocular tuberculosis. Surv Ophthalmol 1993;38:229-56

7 Elliot J. Thirty-year observation of patients with Eales' disease. Am J Ophthalmol 1975;80:404-8

8 Elliot AJ. Recurrent intraocular hemorrhage in young adults (Eales' disease). A report of thirty-one cases. Trans Am Ophthalmol Soc 1954;52:811-75

9 Biswas J, Therese L, Madhavan H. Use of polymerase chain reaction in detection of Mycobacterium tuberculosis comlex DNA from vitreous sample of Eales' disease. Br J Ophthalmol 1999;83:994.

10 Madhavan HN, Therese KL, Gunisha P, et al. Polymerase chain reaction for detection of Mycobacterium tuberculosis in epiretinal membrane in Eales' disease. Invest Ophthalmol Vis Sci 2000;41:822-5.

11 Biswas J, Mukesh BN, Naratin S, et al. Profiling of human leukocyte antigens in Eales' disease. Int Ophthalmol 1998;21:277-81.

12 Rosen PH, Spalton DJ, Graham EM. Intraocular tuberculosis. Eye 1990;4:486-92.

13 Shah SM, Howard RS, Sarkies NJC, et al. Tuberculosis presenting as retinal vasculities. J Roy Soc Med 1988;81:232-3.

14 Magargal LE, Walsh AW, Magargal HO, et al. Treatment of Eales' disease with scatter laser photocoagulation. Ann Ophthalmol 1989;21:300-2.

15 Shanmugam MP, Badrinath SS, Gopal L, et al. long term visual results of vitrectomy for Eales' disease complications. Int Ophthalmol 1998;22:61-4. 